OPEN ACCESS

Edited by:

Adriano Nunes-Nesi,

Universidade Federal de

Viçosa, Brazil

Reviewed by:

Kazuo Nakashima,

Japan International Research Center

for Agricultural Sciences, Japan

Takuya Yoshida,

Max Planck Institute of Molecular

Plant Physiology, Germany

*Correspondence:

Hua Fu

13825164071@139.com

Jun Liu

liujun139@139.com; liujun@gdaas.cn

Specialty section: This article was submitted to

Plant Physiology,

a section of the journal

Frontiers in Plant Science

Received: 18 March 2019

Accepted: 07 June 2019

Published: 27 June 2019

Citation:

Chen B-X, Peng $Y-X$, Gao J-D, Zhang Q, Liu Q-J, Fu H and Liu J (2019) Coumarin-Induced Delay of Rice Seed Germination Is Mediated

by Suppression of Abscisic Acid

Catabolism and Reactive Oxygen Species Production.

Front. Plant Sci. 10:828.

doi: 10.3389/fp/s.2019.00828

\section{Coumarin-Induced Delay of Rice Seed Germination Is Mediated by Suppression of Abscisic Acid Catabolism and Reactive Oxygen Species Production}

\author{
Bing-Xian Chen ${ }^{1}$, Yuan-Xuan Peng ${ }^{1,2}$, Jia-Dong Gao', Qi Zhang ${ }^{1}$, Qin-Jian Liu', \\ Hua Fu${ }^{3 *}$ and Jun Liu ${ }^{1 *}$
}

\begin{abstract}
${ }^{1}$ Argo-Biological Gene Research Center, Guangdong Academy of Agricultural Sciences, Guangzhou, China, ${ }^{2}$ College of Agriculture and Biology, Zhongkai University of Agriculture and Engineering, Guangzhou, China, ${ }^{3}$ Rice Research Institute, Guangdong Academy of Agricultural Sciences, Guangzhou, China
\end{abstract}

Abscisic acid (ABA) is a crucial phytohormone for the regulation of seed germination. The ABA content of seeds is regulated by synthesis and catabolic pathways. Coumarin, an important plant allelochemical, can inhibit seed germination effectively, although whether it is involved in the regulation of ABA content during seed germination has not been elucidated. For the study reported herein, we show that coumarin effectively inhibits rice seed germination and vivipary. We found that the ABA content gradually decreased in water-imbibed rice seeds and that the content and activity of the Oryza sativa 9-cis epoxycarotenoid dioxygenases (OsNCEDs), which are ABA synthases, decreased during seed germination. At the transcription level, the expression of OsNCED1-3 appeared to decrease, whereas the expression of the ABA 8'-hydroxylase 2 and 3 genes (OsABA8'ox2/3) first appeared to increase and then decrease. Samples of rice seeds were also imbibed in water containing coumarin, which increased their ABA content but did not significantly increase the activity or content of their OsNCEDs or OSNCED1-3 transcription. Interestingly, coumarin imbibition remarkably reduced OSABA8'ox2/3 expression in rice embryos, which partially explained how coumarin increased the ABA content of germinating rice embryos. Coumarin also inhibited the accumulation of reactive oxygen species (ROS) in rice embryos and increased the activity of superoxide dismutase and catalase, which are indispensable for seed germination. These results indicate that coumarin delays seed germination by inhibiting ABA catabolism, particularly by decreasing the expression of OsABA8'ox2/3 rather than by increasing ABA synthesis. Moreover, coumarin increases the ABA content while decreasing the ROS content in rice embryos. Our results enhance our understanding of the regulation of $\mathrm{ABA}$ and $\mathrm{ROS}$ during seed germination and provide theoretical support for application of coumarin to prevent sprouting before crop harvesting.

Keywords: seed germination, coumarin, abscisic acid synthesis, abscisic acid catabolism, reactive oxygen species, Oryza sativa, gene expression, enzyme activity 


\section{INTRODUCTION}

Seed germination is a critical step in the life cycle of higher plants. During germination, viable seeds develop into plants upon imbibition in water, which then allows for embryo growth until the radicle breaks through the peripheral tissue structures (endosperm and seed coat; Bewley et al., 2013).

Seed germination is regulated by various factors, including phytohormones, e.g., gibberellin, ethylene, and brassinosteroids, which promote germination, and abscisic acid (ABA), which promotes dormancy (Koornneef et al., 1998; Millar et al., 2006). For rice and Arabidopsis, a decrease in the ABA content of their seeds is necessary for germination (Ali-Rachedi et al., 2004; Zhu et al., 2009). In addition, many studies have shown that certain seed germination inhibitors regulate germination by altering the content of ABA in seeds. For example, glucose and copper inhibit rice seed germination by increasing the amount of ABA (Zhu et al., 2009; Ye et al., 2014). Furthermore, fluridone, a synthetic inhibitor of $A B A$, effectively reduces the ABA content of seeds, which promotes seed germination. Therefore, it is apparent that the ABA content of seeds determines whether germination will occur.

Accumulation of endogenous $\mathrm{ABA}$ in seeds depends on a dynamic balance between ABA synthesis and catabolism. In plants, $A B A$ is synthesized directly from carotenoids, with the committed step being catalyzed by 9-cis epoxycarotenoid dioxygenase (NCED), which cleaves 9-cis xanthophyll to xanthoxin (Nambara and Marion-Poll, 2005; Rodríguez-Gacio et al., 2009). In water-stressed beans, the transcription of the key NCED gene, $P v N C E D 1$, and subsequent translation of its mRNA correlate directly with changes in the endogenous ABA content (Qin and Zeevaart, 1999). For Arabidopsis seeds, NCED mutants germinate more rapidly than do wild-type seeds (Frey et al., 2012), and ectopic expression of the sorghum NCED gene into Arabidopsis caused 9- to 73-fold increases in ABA levels and allowed the seed to remain deeply dormant for over 3 months (Nonogaki et al., 2014). The cytochrome P450, CYP707A2, is an ABA 8'-hydroxylase (ABA8'ox) that inactivates $\mathrm{ABA}$ by chemically modifying it and thereby reduces the $\mathrm{ABA}$ content in seeds and, in turn, relieves seed dormancy and promoting germination. Expression of Arabidopsis thaliana CYP707A2 (AtCYP707A2) in germinating Arabidopsis seeds is associated with a rapid decline in ABA content (Kushiro et al., 2004). In the seeds of a cyp707a2 mutant, its ABA failed to decrease in response to nitrate, which can release seed dormancy in Arabidopsis by reducing ABA levels, and the seeds did not germinate (Matakiadis et al., 2009).

The allelochemical coumarin, an unsaturated lactone, inhibits germination or reduces seedling growth of lettuce (Bewley et al., 2013). It induced dormancy in lettuce seeds by antagonizing gibberellin function, as the addition of gibberellin was unable to reverse coumarin-induced inhibition (Khan and Tolbert, 1966; Berrie et al., 1968). Although coumarin regulates germination, whether it acts by regulating the synthesis and/ or catabolism of $\mathrm{ABA}$ is unclear. Coumarin inhibits catalase, superoxide dismutase (SOD), and ascorbate peroxidase (APX), all of which destroyed reactive oxygen species (ROS) in
Sorghum sudanense seeds and thereby inhibited their germination (Wang et al., 2017) because ROS are needed to loosen the cell wall of seeds necessary for germination (Müller et al., 2009). ABA reduced ROS production during germination of imbibed rice seeds (Ye et al., 2012), but the relationship among coumarin, ABA synthesis/catabolism, and ROS production with respect to inhibition of germination is unclear.

Although the physiological and molecular mechanisms of seed dormancy and germination of $A$. thaliana seeds have been well studied, there are many unresolved issues concerning cultivation of crops in the field. How to effectively solve such issues, e.g., slow germination rates and pre-harvest sprouting (PHS), is crucial to increasing agricultural yields (Bewley et al., 2013; Nonogaki et al., 2018). We previously found that coumarin can effectively prevent rice PHS, but the physiological and molecular mechanisms involved are still unclear. For the study reported herein, we comprehensively examined the effects of coumarin on germination, ABA synthesis and its degradation, ROS production, and expression of related enzyme genes in rice seeds. We found that coumarin inhibited degradation of ABA by inhibiting OsABA8'ox activity, rather than ABA synthesis. Our results help clarify the relationship between coumarin, $\mathrm{ABA}$, and ROS during seed germination. Our results also suggest that coumarin could be used to inhibit or prevent PHS in crops in the field.

\section{MATERIALS AND METHODS}

\section{Non-plant Materials}

Coumarin, fluridone, diniconazole, $\mathrm{ABA}$, nitroblue tetrazolium (NBT), 3,3'-diaminobenzidine hydrochloride (DAB), 3,3',5,5'-tetramethylbenzidine (TMB), and 2,3,5-triphenyl tetrazolium chloride (TTC) were purchased from Sigma-Aldrich (USA). Water was doubly distilled before use.

\section{Plant Materials, Seed Germination, and Rice Pre-harvest Sprouting Tests}

Rice seeds (O. sativa ssp. indica cv. R998) with their glume intact were placed into transparent plastic germination boxes $(12 \mathrm{~cm} \times 12 \mathrm{~cm} \times 6 \mathrm{~cm})$ containing two layers of filter paper soaked in water and with one of the treatments shown in Supplementary Figure S1. The final volume of each sample was $25 \mathrm{ml}$. The seeds were incubated in a growth chamber at $28 \pm 1{ }^{\circ} \mathrm{C}$ under a 16 -h light/8-h dark photocycle. The light intensity was $190 \mu \mathrm{mol} / \mathrm{m}^{2} / \mathrm{s}$ by photosynthetic photon flux density. Seeds were counted at 3 -h intervals between 36 and $48 \mathrm{~h}$.

To test for rice vivipary, seeds were soaked in water containing $1,5,10$, or $20 \mathrm{mM}$ coumarin for $1 \mathrm{~min}$, then removed and allowed to dry at room temperature before being imbibed in water to determine the percentage of seeds that would germinate. And then a suitable concentration of aqueous coumarin that was found to effectively inhibit germination, i.e., $10 \mathrm{mM}$ coumarin, was sprayed onto mature seeds on rice plants (15 days before harvest) that had been growing in a greenhouse. The control group (mature seeds on rice plants) was sprayed with water. 
The temperature and humidity in the greenhouse were kept at $38 \pm 2{ }^{\circ} \mathrm{C}$ and $95 \pm 2 \%$. The treated rice plants were harvested 15 days after being sprayed.

Seeds with protruding radicles were regarded as having completed germination. The number of germinated seeds at each time point was converted to a percentage, and the mean value \pm SE of three biological replicates of 100 seeds each was calculated. Seeds were photographed using a stereomicroscope (SteREO Lumar V12, Zeiss, Germany).

\section{Measurement of Endogenous Abscisic Acid Levels}

Rice seed embryos ( $1 \mathrm{~g}$ ) were ground into a powder in liquid nitrogen, and $10 \mathrm{ml}$ of isopropanol/hydrochloric acid (3:1) containing $8 \mu \mathrm{l}$ of $50 \mathrm{ng} / \mathrm{ml} \mathrm{ABA}$ as an internal standard was added into the powder, and the mixture was then shaken at $4^{\circ} \mathrm{C}$ for $30 \mathrm{~min}$. Next, $20 \mathrm{ml}$ of dichloromethane was added into the mixture, which was then shaken at $4^{\circ} \mathrm{C}$ for $30 \mathrm{~min}$. The mixture was centrifuged at $11,255 \times g$ for $5 \mathrm{~min}$ at $4^{\circ} \mathrm{C}$, after which the upper portion of the supernatant was discarded and the lower organic phase retained. The organic phase was dried under nitrogen in the dark and then dissolved in $400 \mu \mathrm{l}$ of methanol containing $0.1 \%$ formic acid. The solution was passed through a $0.22-\mu \mathrm{m}$ filter, and the ABA content was determined with HPLC (Model 1,290, Agilent, USA) coupled with tandem mass spectrometry (MS/MS, SCIEX-6500Qtrap, $\mathrm{AB}$ SCIEX, USA). The mean value $\pm \mathrm{SE}$ of three biological replicates is reported.

\section{9-Cis Epoxycarotenoid Dioxygenase Content and Activity Assays}

Phosphate-buffered saline ( $\mathrm{pH} 7.4,9 \mathrm{ml}$ ) was added into $1 \mathrm{~g}$ of embryos, which were then homogenized by grinding with a mortar and pestle. The homogenate was centrifuged at $600 \times g$ and $4^{\circ} \mathrm{C}$ for $5 \mathrm{~min}$, after which the supernatant was retained. The sample was then subjected to ELISA using reagents from a Plant NCED ELISA kit according to the manufacturer's instructions (Beijing Chenglin Biotechnology Co., Ltd., Beijing, China). The $A_{450}$ value of the labeled antibody-antigen complex was measured using a full-wavelength ELASA instrument (Varioskan Flash, Thermo, USA) to determine the concentration and activity of NCED. The mean value \pm SE of three biological replicates was calculated.

\section{Phylogenetic Relationships and Structural Domain Analysis}

To better understand the evolution of the NCED and ABA8'ox gene families in monocot and eudicot, we generated unrooted maximum-likelihood phylogenetic trees. We selected ABA metabolism-related genes from rice Nipponbare including OsNCED1 (AY838897), OsNCED2 (AY838898), OsNCED3 (AY838899), OsNCED4 (AY838900), OsNCED5 (AY838901), OsABA8'ox1 (i.e., OsCYP707A5, AB277270), OsABA8'ox2 (i.e., OsCYP707A6, NM_001068556), and OsABA8'ox3 (i.e., OsCYP707A7, NM_001069901) according to the previous studies (Saika et al., 2007; Zhu et al., 2009; Liu et al., 2011).
In addition, NCED and ABA8'ox genes of other plant species are also included according to published reports (Supplementary Table S1). The sequences of O. sativa, A. thaliana, and other plant species were acquired from the Rice Annotation Project Database, the Arabidopsis Information Resource (TAIR v10.02), or NCBI. Alignment of sequences and phylogenetic analysis were carried out by using Clustal W with default parameters and MEGA7 with maximum-likelihood method. Finally, the phylogenetic tree was perfected by ITOL $^{1}$. The Pfam and SMART databases ${ }^{2}$ were used to analyze the functional domains of the identified NCED and ABA8'ox candidates described by Chang et al. (2016).

\section{In silico Expression Profiles (Heat Maps) and Quantitative Polymerase Chain Reaction of 9-Cis Epoxycarotenoid Dioxygenase and ABA8'ox mRNAs}

We used the Os_51k microarray data in the Genevestigator V3 database to analyze the expression profiles of rice NCED and $\mathrm{ABA} 8$ 'ox genes by constructing heat maps from the datasets (Hruz et al., 2008).

To characterize the expression profiles of the OsNCED and OsABA8'ox genes by quantitative polymerase chain reaction (qPCR), 30 embryos from seeds that had been incubated in water or $1 \mathrm{mM}$ coumarin for six imbibition times $(6,12,18$, 24,36 , or $48 \mathrm{~h}$ ) were extracted and immediately frozen at $-80^{\circ} \mathrm{C}$. Total RNA was isolated using Column Plant RNAout 2.0 kit reagents (TIANDZ, China), and qPCR was performed as described (Chen et al., 2016). The gene-specific primers (Supplementary Table S2) were designed to avoid conserved regions, introns, and the single exon-exon junction. OsGAPDH1 mRNA (RAP-DB ID: Os02g0601300) expression served as the internal control. The mean value $\pm \mathrm{SE}$ for each experiment of three biological replicates was calculated.

\section{Histochemical Localization of $\mathrm{O}_{2}^{-}$and $\mathrm{H}_{2} \mathrm{O}_{2}$} We used NBT and DAB, respectively, to stain seeds for $\mathrm{O}_{2}{ }^{-}$ and $\mathrm{H}_{2} \mathrm{O}_{2}$ as described (Li et al., 2017). After rice seeds had imbibed water or $1 \mathrm{mM}$ coumarin for 6,18 , or $36 \mathrm{~h}$, five whole seeds and five half-granule seeds each containing an embryo were removed and incubated with $1 \mathrm{mM} \mathrm{NBT}$ in $10 \mathrm{mM}$ Tris- $\mathrm{HCl}(\mathrm{pH} \mathrm{7.0)}$ or $1 \mathrm{mg} / \mathrm{ml} \mathrm{DAB}(\mathrm{pH} \mathrm{3.8)}$ ) at room temperature for $30 \mathrm{~min}$, then washed with double-distilled water, and photographed under a SteREO Lumar V12 stereomicroscope.

\section{TTC Staining}

TTC (0.5 g) was dissolved in $2 \mathrm{ml}$ of ethanol, which was then added into $100 \mathrm{ml}$ of double-distilled water, and stored at $4^{\circ} \mathrm{C}$ in the dark until used. After incubation in water or $1 \mathrm{mM}$ coumarin for 6,18 , or $36 \mathrm{~h}$, five whole seeds and five half-granule seeds each containing an embryo were stained

${ }^{1}$ https://itol.embl.de/

${ }^{2}$ http://smart.embl-heidelberg.de/ 
with $0.5 \%$ TTC at $35^{\circ} \mathrm{C}$ for $3 \mathrm{~h}$, then washed three times with water, and photographed as described above.

\section{Histochemical Detection of Peroxidase Activity}

Peroxidase is the key enzyme for production of $\bullet \mathrm{OH}$, and the level of peroxidase activity indirectly reflects the production and accumulation of $\bullet \mathrm{OH}$. We detected peroxidase activity histochemically by TMB staining as described (Li et al., 2017). Briefly, after rice seeds had been imbibed in water or $1 \mathrm{mM}$ coumarin for 6,18 , or $36 \mathrm{~h}$, five whole seeds and five halfgranule seeds (each containing an embryo) were incubated in $0.2 \% \mathrm{TMB}, 1 \mathrm{mM} \mathrm{H}_{2} \mathrm{O}_{2}, 20 \mathrm{mM}$ potassium phosphate $(\mathrm{pH}$ 6.5) at room temperature for $30 \mathrm{~min}$, then washed in water and photographed as described above.

\section{Superoxide Dismutase, Catalase, and Ascorbate Peroxidase Activity Measurements}

A crude extract of the enzymes from rice seed embryos was prepared according to Ye et al. (2014). Frozen seeds (0.5 g) were homogenized on ice with $1 \mathrm{ml}$ of $50 \mathrm{mM}$ potassium phosphate ( $\mathrm{pH} 7.0$ ), $1 \mathrm{mM}$ EDTA, and 1\% polyvinylpyrrolidone. Each homogenate was centrifuged at $12,000 \times g$ for $30 \mathrm{~min}$ at $4^{\circ} \mathrm{C}$, and each supernatant was retained for enzyme assays. Protein content was determined according to the method of Bradford (1976) with bovine serum albumin as the standard. The activities of SOD, catalase, and APX were determined spectrophotometrically according to Ye et al. (2014). The mean value \pm SE for each experiment of three biological replicates was calculated.

\section{Statistical Analysis}

Data are presented as the mean \pm SE of three replicates. One-way analysis of variance was used to compare mean values, and when significant, differences between individual means were compared with the Fisher's least-significant difference test. Student's $t$ test was conducted to evaluate variances in the changes in germination percentage, $\mathrm{ABA}$ content, and expression levels of OsNCED1-3 and OsABA2/3.

\section{RESULTS}

\section{Germination of Rice Seeds Is Inhibited by Coumarin and by Inhibitors of Abscisic Acid Catabolism but not by Inhibitors of Abscisic Acid Synthesis}

A relatively small dose of coumarin can inhibit the germination of lettuce (Berrie et al., 1968), soybean (Colpas et al., 2003), and Sorghum sudanense seeds (Wang et al., 2017). We soaked rice seeds in $1,5,10$, or $20 \mathrm{mM}$ coumarin for $1 \mathrm{~min}$ and then transferred them into water for germination. Only the seeds treated with 1 or $5 \mathrm{mM}$ coumarin germinated, but their progression to the seedling stage was inhibited compared with control seeds. Conversely, seeds treated with 10 or $20 \mathrm{mM}$ coumarin were unable to germinate (Figure 1A). In the greenhouse, we mimicked the high-temperature and highhumidity conditions for field-grown rice at the pre-harvest stage. When we sprayed the rice with $10 \mathrm{mM}$ coumarin, their vivipary was inhibited $(2.8 \%$ vs. $35.4 \%$ for the water-sprayed controls; Figure 1B).

To further investigate the effect(s) of different concentrations of coumarin on rice seed germination, we carried out a germination time-course experiment, which revealed that rice seeds began to germinate at $36 \mathrm{~h}$ when imbibed in water. Conversely, exposure to coumarin inhibited rice seed germination, and this inhibition was more obvious as the coumarin concentration increased. Notably, 1 or $2 \mathrm{mM}$ coumarin decreased the number of seeds that germinated throughout the experiment, i.e., at each time point, and delayed the initial germination time by 3 and 9 h, respectively. After $72 \mathrm{~h}$, the germination percentages for seeds exposed to 1 or $2 \mathrm{mM}$ coumarin were 83 and $53 \%$, respectively, of the control seeds (Figure 2).

Fluridone is a potent inhibitor of ABA synthesis (Nambara and Marion-Poll, 2005; Martínez-Andújar et al., 2011), and diniconazole is a potent inhibitor of ABA catabolism (Kitahata et al., 2005). Fluridone $(50 \mu \mathrm{M})$ increased rice seed germination by $\sim 8 \%$ at $48 \mathrm{~h}$ and by $\sim 9 \%$ at $60 \mathrm{~h}$. Diniconazole $(10 \mu \mathrm{M})$ decreased rice seed germination by $12 \%$ at $48 \mathrm{~h}$ and $9 \%$ at $60 \mathrm{~h}$ (Supplementary Figure S1). When rice seeds were imbibed in $0.1,0.5,1$, or $2 \mathrm{mM}$ coumarin plus $50 \mu \mathrm{M}$ fluridone, seed germination was not significantly altered compared with seeds imbibed only in coumarin. Conversely, germination was further inhibited when the seeds were imbibed in $2 \mathrm{mM}$ coumarin plus $10 \mu \mathrm{M}$ diniconazole (5\% germination) compared with coumarin alone (10\% germination; Supplementary Figure S1). These results suggested that coumarin-mediated inhibition of germination and fluridonemediated stimulation of germination may not offset each other, whereas coumarin and diniconazole may act synergistically to inhibit germination.

\section{Coumarin Significantly Increases the Abscisic Acid Content of Rice Embryos but Does not Substantially Affect 9-Cis Epoxycarotenoid Dioxygenase Content or Activity}

During imbibition of water by Arabidopsis and barley seeds, the ABA content gradually decreases (Millar et al., 2006). To determine ABA levels in rice seeds, we first isolated embryos from germinating seeds that had imbibed water or $0.5,1$, or $2 \mathrm{mM}$ coumarin for 6 to $48 \mathrm{~h}$. After which, the ABA concentrations in the embryos were measured. For the waterimbibed seeds, the ABA content decreased rapidly between 6 and $18 \mathrm{~h}$ and slowed thereafter (Figure 3). The ABA content decreased slightly in seeds imbibed in $0.5 \mathrm{mM}$ coumarin, and these seeds had an ABA-content time course similar to that of the water-imbibed seeds. Conversely, imbibition of 1 or $2 \mathrm{mM}$ coumarin substantially enhanced the relative ABA content of the seeds at all time points in comparison with that of the 

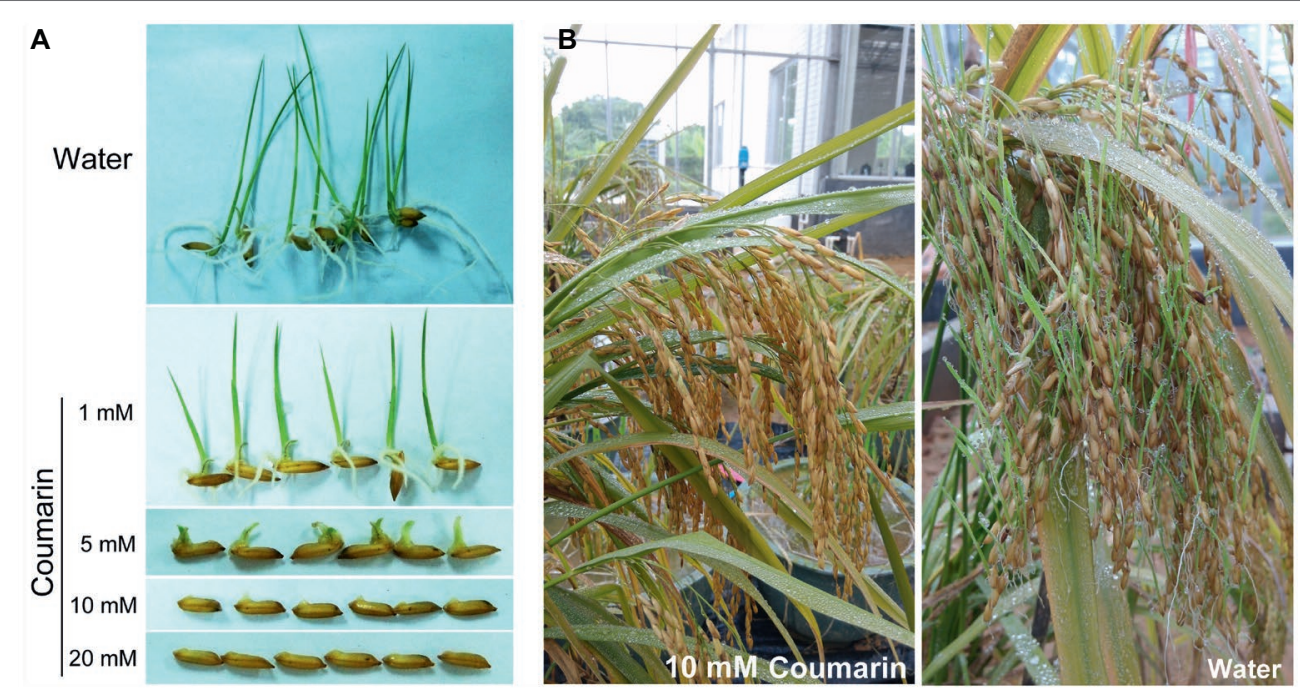

FIGURE 1 | Morphologies of germinating seeds and pre-harvest-sprouted rice. (A) Morphologies of rice seeds imbibed in water or in 1, 5, 10, or $20 \mathrm{mM}$ coumarin. (B) The extent of pre-harvest-sprouted rice sprayed with $10 \mathrm{mM}$ coumarin (left) or water (right) under greenhouse conditions of $38 \pm 2^{\circ} \mathrm{C}$ and $95 \pm 2 \%$ humidity.

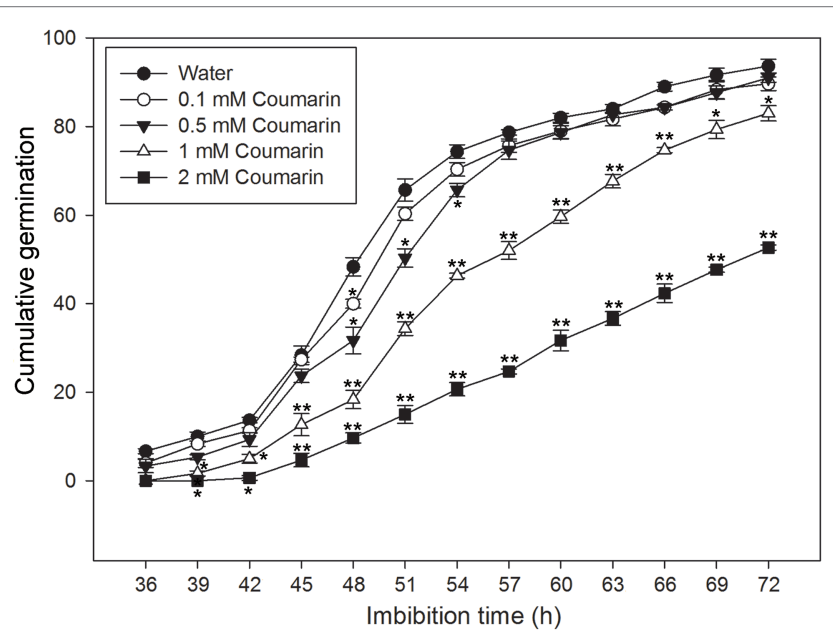

FIGURE 2 | Germination time courses for rice seeds imbibed in water, or in $0.1,0.5,1$, or $2 \mathrm{mM}$ coumarin. The number of seeds that had germinated was determined every $3 \mathrm{~h}$ for $72 \mathrm{~h}$, and the results are presented as the cumulative germination percentage. Data represent the mean \pm SE of three biological replicates of 100 seeds each. Significant differences in the data of germination percentage in $0.1,0.5,1$, and $2 \mathrm{mM}$ coumarin from those in water at each imbibition time point were assessed by Student's $t$ test $\left({ }^{*} p<0.05\right.$; $\left.{ }^{* *} p<0.01\right)$.

water-imbibed seeds, although the ABA content of the seeds that had imbibed 1 or $2 \mathrm{mM}$ coumarin also gradually and continuously decreased except for the 12-h time point of seeds imbibed with $2 \mathrm{mM}$ coumarin (Figure 3).

NCED is the key enzyme in ABA synthesis, and changes in NCED activity are closely related to the ABA content of plant seeds (Yamaguchi et al., 2007). An ELISA study was performed to explore changes in the NCED level during the production of $\mathrm{ABA}$ in rice embryos. For embryos of rice seeds imbibed in water, both the NCED content and activity remained at relatively high levels from 6 to 24 or $18 \mathrm{~h}$ and then remarkably decreased afterward, and similar results were obtained for seeds imbibed in coumarin (Figures 4A,B). However, $0.5,1$, or $2 \mathrm{mM}$ coumarin did not substantially promote or inhibit the NCED content during the whole imbibition process (Figures 4A,B).

\section{Identification and Phylogenetic Analysis of the Rice 9-Cis Epoxycarotenoid Dioxygenase and ABA8'ox Genes}

To identify the NCED and ABA8'ox genes present in rice, we used the Arabidopsis NCED and CYP707A sequences as queries for searches of the rice genome database. For multiplesequence alignment, we also included NCED and ABA8'ox sequences from seven other plant species (Figure 5). All NCED and ABA8'ox sequences included all their domains, e.g., an RPE65 domain and a p450 domain, for the NCED and ABA8'ox sequences, respectively (Figure 5). To investigate the evolutionary relationships among these proteins, we generated two unrooted phylogenetic trees one containing the NCED sequences and the other containing the ABA8'ox sequences from the aforementioned species. According to the topological structures of the trees, the NCED and the ABA8'ox families of monocotyledon and dicotyledon plants can each be divided into three subfamilies, denoted Sub I, II, and III. For both enzymes, those in the Sub I family are found in monocotyledonous and dicotyledonous plants, whereas those in Sub II and III families belong to monocotyledons or dicotyledons, respectively (Figure 5). The OsNCED1/2 sequences are in Sub I, whereas the OsNCED3-5 sequences are in the monocotyledon-specific Sub II family (Figure 5A). The OsABA8'ox $2 / 3$ sequences belong to the Sub I family, whereas the OsABA8'ox1 sequence is in the monocotyledon-specific Sub II family (Figure 5B). These results suggested that, among monocotyledonous and dicotyledonous plants, each of the NCED and ABA8'ox families 


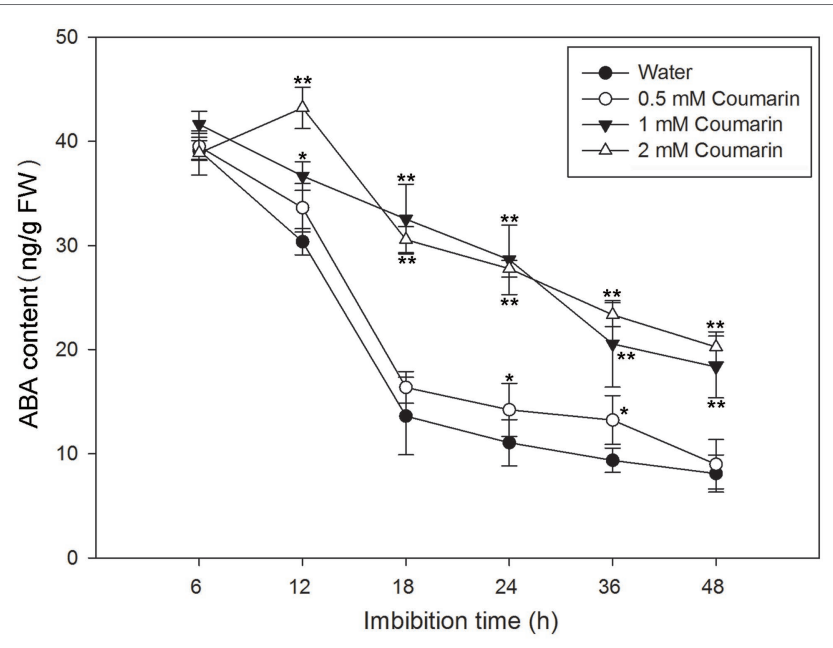

FIGURE 3 | Changes in ABA levels in imbibed seeds in the absence or presence of coumarin. Seeds were imbibed at $28^{\circ} \mathrm{C}$ in water, or in $0.5,1$, or $2 \mathrm{mM}$ coumarin. Seed samples were collected at different time points and stored at $-80^{\circ} \mathrm{C}$ for $\mathrm{ABA}$ content determination. ABA was quantified with HPLC. Data represent the mean \pm SE of three biological replicates each of $1 \mathrm{~g}$ of embryos. Significant differences in the data of ABA content in 0.5, 1, and $2 \mathrm{mM}$ coumarin from those in water at each imbibition time point were assessed by Student's $t$ test $\left({ }^{\star} p<0.05 ;{ }^{\star \star} p<0.01\right)$. FW, fresh weight.

has evolved from a single (but different) ancestor. During the evolution of monocotyledons and dicotyledons, these gene families diverged with genetic duplication to generate their own distinct subfamilies.

\section{The Expression Profiles of OsNCED and OsABA8'ox Genes Differed Substantially During Rice Germination, and the Transcript Levels of OsABA8'oxs in Rice Embryos Decrease After Imbibition With Coumarin}

We examined the expression patterns of OsNCED1-5 and $O s A B A 8^{\prime} \circ \times 1-3$ by displaying the rice microarray data in the Genevestigator database as heat maps. We found substantial differences in their expression during different development stages of rice (Supplementary Figure S2). In seedlings, OsNCED3 and $O s A B A 8^{\prime} \circ x 3$ were each expressed at a higher level than those of the other genes, suggesting that these two genes may be involved in seed germination and establishment of seedlings (Supplementary Figure S2A). In comparison with the individual expression the OsNCED genes, except for OsNCED3, expression of OsABA8'ox $2 / 3$ was greater, and the expression of $O s A B A 8^{\prime} \circ \times 2 / 3$ decreased gradually as germination progressed (Supplementary Figure S2B).

Next, qPCR was used to further characterize the expression profiles of OsNCED1-5 and OsABA8'ox1-3. The results were partially consistent with the expression patterns that were based on the microarray data. The discrepancies may be a consequence of different rice varieties and/or materials. OsNCED1-3 were expressed at higher levels than OsNCED4-5, and $O s A B A 8^{\prime} \circ \times 2 / 3$ were expressed at higher levels than
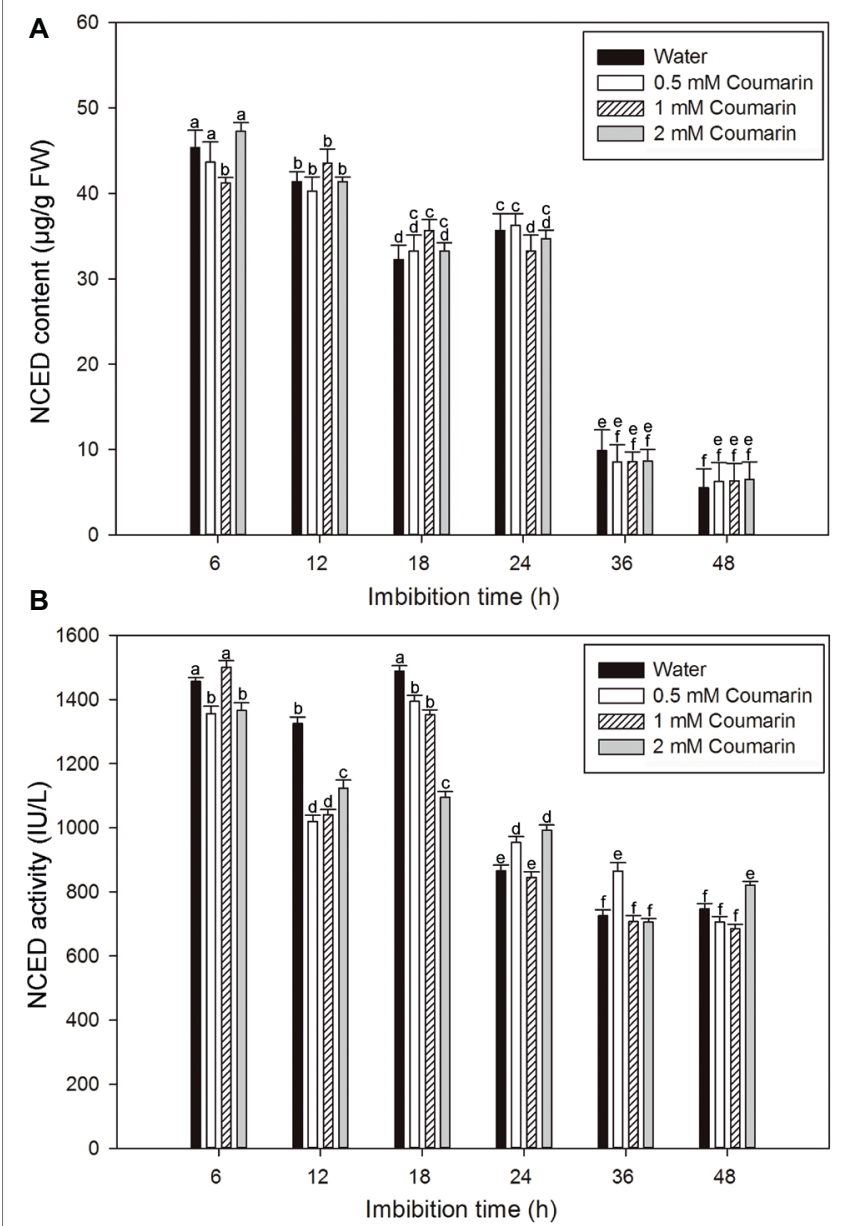

FIGURE 4 | Changes in NCED content and activity in imbibed seeds in the absence or presence of coumarin. Seeds were imbibed at $28^{\circ} \mathrm{C}$ in water, or in 0.5 , 1 , or $2 \mathrm{mM}$ coumarin. Seed samples were collected at different times and stored at $-80^{\circ} \mathrm{C}$ for NCED content (A) and NCED activity (B) determination. NCED content and activity were determined ELISA. Data represent the mean \pm SE of three biological replicates each of $1 \mathrm{~g}$ of embryos. Means denoted by the same letter did not significantly differ at $p<0.05$ according to Fisher's least significant difference test.

OsABA8'ox1 (Figure 6A). Therefore, the expression profiles of OsNCED1-3 and OsABA8'ox2/3 were then examined throughout the course of germination (at 6, 12, 18, 24, 36, and $48 \mathrm{~h}$ ) by qPCR when seed was imbibed in water and $1 \mathrm{mM}$ coumarin. The expression of OsNCED1/3 initially increased, peaked between 12 and $18 \mathrm{~h}$, and then decreased gradually (Figures 6B,D). The expression of OsNCED2 began to gradually decrease at $6 \mathrm{~h}$ and reached its lowest value at $48 \mathrm{~h}$ of imbibition (Figure 6C). Notably, $1 \mathrm{mM}$ coumarin had little consistent effect on the expression of OsNCED1-3, as it inhibited or enhanced their expression at specific time points. The expression patterns of $O s A B A 8^{\prime} \circ x 2 / 3$ were similar, showing first an increase and then a decrease, with a peak value at $18 \mathrm{~h}$ for OsABA8'ox2 and at $12 \mathrm{~h}$ for OsABA8'ox3. Interestingly, $1 \mathrm{mM}$ coumarin strongly inhibited the transcription of both genes, particularly that of $O s A B A 8^{\prime} \circ \times 2$ 


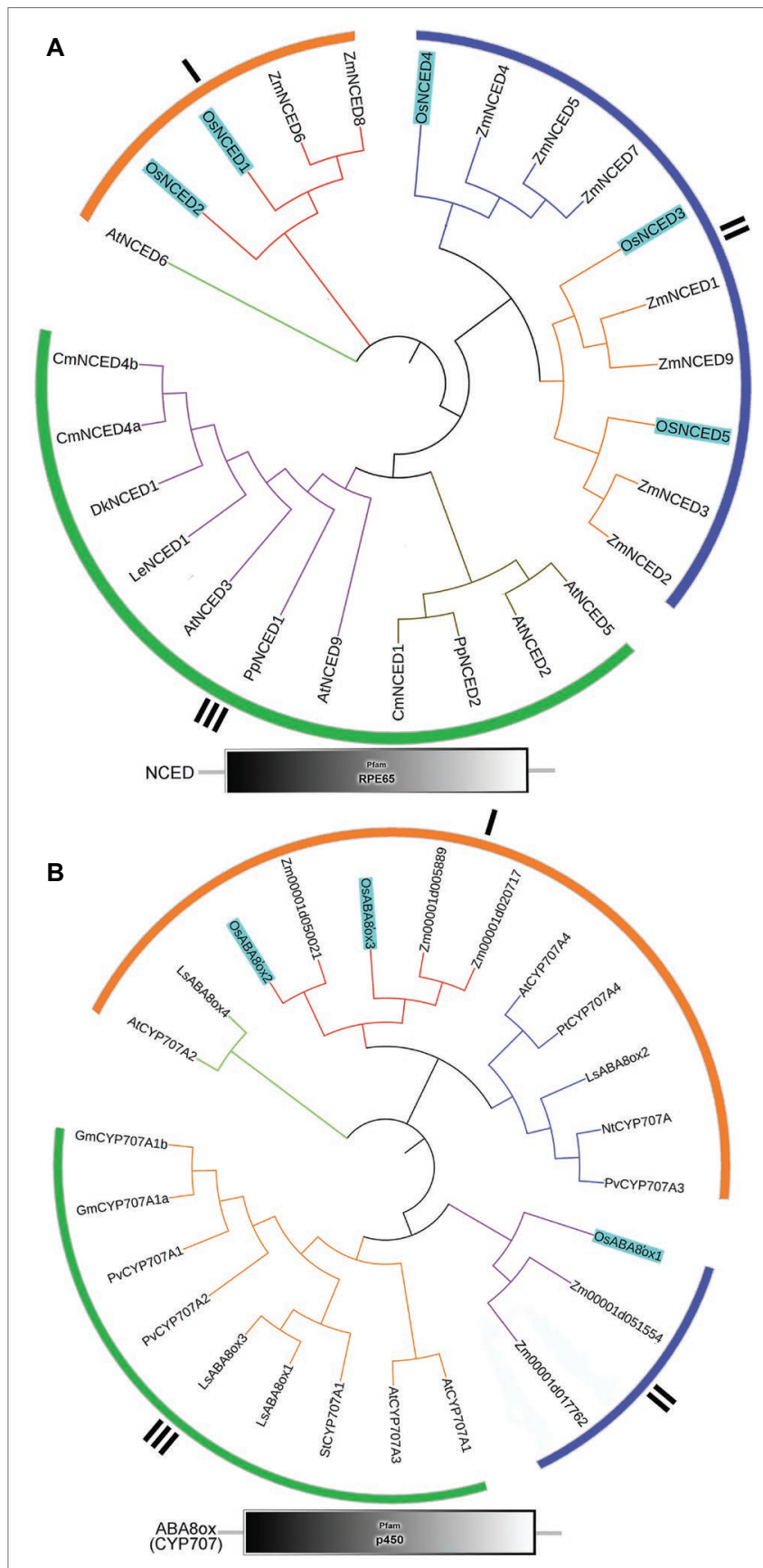

FIGURE 5 | Phylogenetic trees and predicted domains for the NCED and ABA8'ox families. (A) An unrooted maximum-likelihood phylogenetic tree based on an alignment of the amino oxidase sequences of NCEDs from the following plant species: O. sativa, A. thaliana, Zea mays, Solanum lycopersicum, Prunus persica, Diospyros kaki, Cucumis melo, and Chrysanthemum $\times$ morifolium. The sequences used to build the tree and their GenBank accession numbers are displayed in Supplementary Table S1. Domain organization of the NCEDs from the aforementioned plants is shown below the tree. (B) An unrooted maximum-likelihood phylogenetic tree based on the alignment of the amino oxidase sequences of OsABA8'oxs from the following plant species: O. sativa, A. thaliana, Zea mays, Populus trichocarpa, Lactuca sativa, Glycine max, Nicotiana tabacum, Solanum tuberosum, and Phaseolus vulgaris. The sequences used to build the tree and their GenBank accession numbers are displayed in Supplementary Table S1. Domain organization of ABA8'oxs in the abovementioned plants is shown below the tree. between 6 and $24 \mathrm{~h}$ (2.3-3.4-fold) and that of OsABA8'ox3 at $12 \mathrm{~h}$ (1.5-fold; Figures $6 \mathrm{E}, \mathrm{F})$.

\section{Coumarin Has a Negligible Effect on Seed Viability but Suppresses Accumulation of Endogenous Reactive Oxygen Species and Enhances the Activities of Reactive Oxygen Species-Degrading Enzymes}

TTC staining is an effective method for determining seed viability. Seeds that are hardy with respect to viability also tend to undergo germination (Batty et al., 2001). When lettuce seeds are chemically poisoned by sodium dichloroisocyanurate, their viability declines and their germination potential is expected to be inhibited (Zhang et al., 2014). To investigate the effect of coumarin on seed viability, we stained seeds imbibed in water or coumarin with a TTC solution and found that the coumarin-treated seeds were stained to a similar extent as those imbibed in water (Figure 7A), suggesting that although coumarin inhibits seed germination, its mechanism does not involve reducing seed viability.

ROS are critical for seed germination. We used NBT and DAB staining to detect $\mathrm{O}_{2}^{-}$and $\mathrm{H}_{2} \mathrm{O}_{2}$ accumulation during rice seed germination. The activity of peroxidase, which can catalyze the conversion of $\mathrm{O}_{2}^{-}$and $\mathrm{H}_{2} \mathrm{O}_{2}$ to $\bullet \mathrm{OH}$ in seeds, was detected by TMB staining (Müller et al., 2007), thereby indirectly reflecting the accumulation of $\bullet \mathrm{OH}$ (Zhang et al., 2014). When rice seeds were imbibed in water, the accumulation of $\mathrm{O}_{2}^{-}$and $\mathrm{H}_{2} \mathrm{O}_{2}$ in their embryos gradually increased during germination, especially by $36 \mathrm{~h}$ when the first seed had completed germination; by comparison, the embryos from seeds imbibed in coumarin were much less intensely stained (Figures 7B,C), indicating that coumarin had an inhibitory effect on the production of $\mathrm{O}_{2}^{-}$and $\mathrm{H}_{2} \mathrm{O}_{2}$. Conversely, no substantial change in peroxide activity was found for the embryos from the water- and coumarin-imbibed seeds during the germination process, indicating that their $\cdot \mathrm{OH}$ content was not substantially affected by the imbibition process and coumarin (Figure 7D).

Many antioxidant enzymes, e.g., SOD, catalase, and APX play key roles in scavenging ROS in plants (Ye et al., 2014). The results shown in Figure 7 indicated that coumarin inhibited the accumulation of ROS in seeds, but what is its effect(s) on ROS-degrading enzymes? To answer this question, we examined the activities of ROS-scavenging enzymes, i.e., catalase, SOD, and APX, in imbibing seeds. The activities of catalase and APX gradually increased and peaked at $48 \mathrm{~h}$, whereas the activity of SOD gradually decreased during germination of seeds imbibed in water. For seeds imbibed in the presence of coumarin, the SOD and catalase activities increased, especially between 24 and $48 \mathrm{~h}$ of germination, but the APX activity in those seeds was essentially unchanged compared with that of water-imbibed seeds (Figure 8). The enhanced SOD and catalase activities found for embryos from coumarin-imbibed seeds correlated with the inhibition of ROS accumulation found for those embryos. 

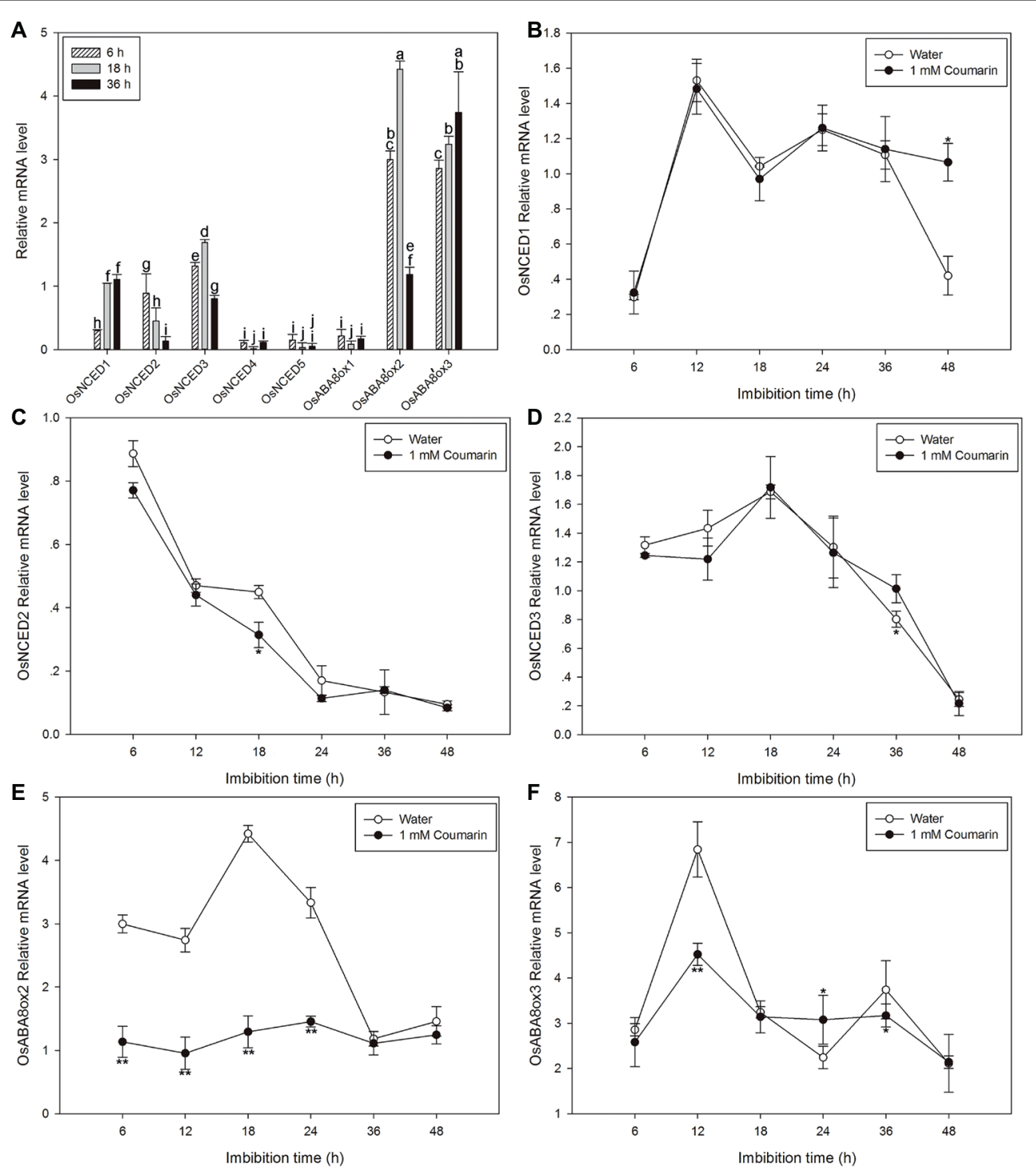

FIGURE 6 | Changes in the expression profiles for OsNCED and OsABA8'ox genes during rice seed germination as assessed with qPCR. (A) The expression levels of OSNCED1-5 and OSABA8'OX1-3 in embryos assayed by qPCR after their seeds had been imbibed in water for 6, 18, or $36 \mathrm{~h}$. Data represent the mean $\pm \mathrm{SE}$ of three biological replicates, each with 30 embryos (0.1 g per replicate). Expression patterns for OsNCED1 (B), OsNCED2 (C), OsNCED3 (D), OsABA8'Ox2 (E), and OSABA8'OX3 (F) in embryos as assayed by qPCR after seed imbibition of water or $1 \mathrm{mM}$ coumarin at $6,12,18,24,36$, and $48 \mathrm{~h}$. Data represent the mean \pm SE of three biological replicates, each with 30 embryos ( $0.1 \mathrm{~g}$ per replicate). Means denoted by the same letter did not significantly differ at $p<0.05$ according to Fisher's least significant difference test (A). Significant differences in the data of relative mRNA level in $1 \mathrm{mM}$ coumarin from those in water at each imbibition time point were assessed by Student's $t$ test $\left(\mathbf{B}-\mathbf{F},{ }^{*} p<0.05 ;{ }^{* *} p<0.01\right)$.

\section{DISCUSSION}

\section{Both Abscisic Acid Synthesis and Catabolism Play Essential Role for Seeds Germinating in Water, Whereas Abscisic Acid Catabolism May Be More Important for Seeds Germinating in Coumarin}

The ABA content in seeds is regulated by the relative rates of its synthesis and degradation. In corn seeds, when phytoene dehydrogenase or NCED activity in the synthesis pathway is inhibited, the seed will sprout prior to harvest
(Robichaud et al., 1980; Durantini et al., 2008). Arabidopsis seeds from cyp707a2, a CYP707A2-type mutant that degrades ABA slowly, showed a delayed germination phenotype (Kushiro et al., 2004; Millar et al., 2006). We found that fluridone (an inhibitor of ABA synthesis) and diniconazole (an inhibitor of ABA degradation) promoted and inhibited germination, respectively, to a similar extent (Supplementary Figure S1). In combination with the result of changes in ABA content of seeds that gradually decreased at the beginning of the imbibition (Figure 3), it seems that a decrease in the ABA content of the seeds was a prerequisite for germination. These findings 

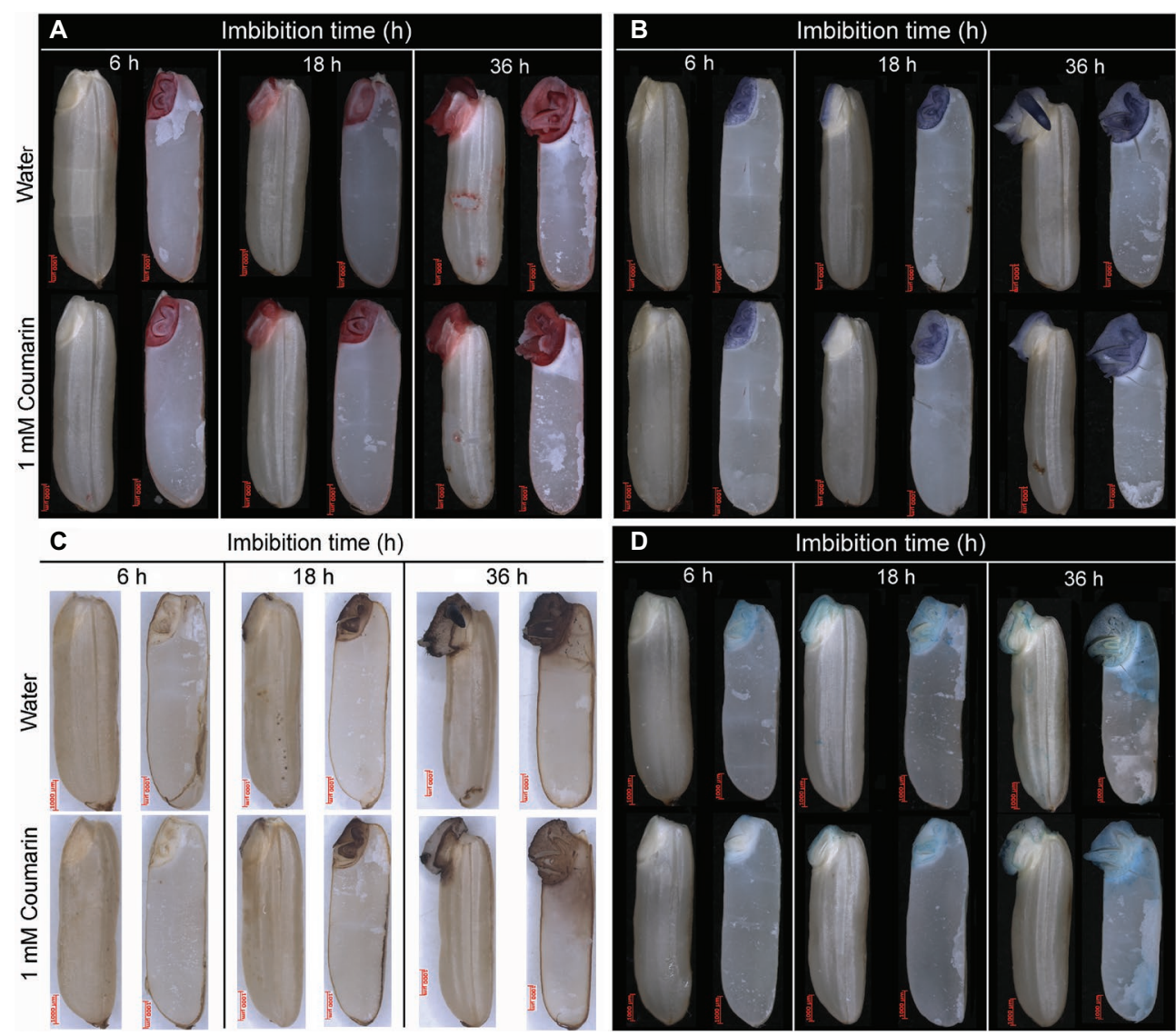

FIGURE 7 | Histochemical staining to assess seed viability, $\mathrm{O}_{2}^{-}$and $\mathrm{H}_{2} \mathrm{O}_{2}$ content, and peroxidase activity during germination of rice seeds in water or coumarin. Seeds were imbibed in water or $1 \mathrm{mM}$ coumarin for 6,18 , or $36 \mathrm{~h}$, after which whole seeds and slit half seeds were stained. (A) Seed viability was assessed by staining with TTC. (B) $\mathrm{O}_{2}^{-}$content was assessed by staining with NBT. (C) $\mathrm{H}_{2} \mathrm{O}_{2}$ content was assessed by staining with DAB. (D) Peroxidase activity was assessed by staining with $\mathrm{TMB}$.

are similar to those found for maize and Arabidopsis (Millar et al., 2006; Durantini et al., 2008). For the expression profiles of OsNCED1-5, OsNCED2/3 were highly expressed and gradually decreased from 18 to $48 \mathrm{~h}$ and that the changes in the expression of those two genes were consistent with the changes in NCED activity and ABA content. Conversely, the expression of $O s A B A 8^{\prime} \circ \times 2 / 3$ gradually increased during early seed imbibition, peaked at 18 and $12 \mathrm{~h}$, respectively, and then decreased (Figures 7E,F). These results may explain the changes in ABA content, as substantial OsABA8'ox activity is needed initially during seed imbibition for rapid ABA degradation, which prepares seeds for germination.

The content of ABA in plants is controlled by ABA biosynthesis and catabolism, which are, in turn, affected by environmental factors such as water availability, temperature, and light exposure (Nambara and Marion-Poll, 2005). Glucose, mannitol, and copper can effectively increase the ABA content of water-imbibed rice seeds (Zhu et al., 2009; Ye et al., 2014). However, the effect of coumarin on ABA synthesis and catabolism during seed germination had seldom been investigated prior to this report. We found that coumarin had a direct dose-dependent effect on germination inhibition (Figure 2), which was consistent with our finding that the dose-dependent effect of coumarin on the ABA content of seeds was also direct (Figure 3). If coumarin inhibits germination by increasing $\mathrm{ABA}$ synthesis, which would increase ABA content in the absence of increased degradation, then it might be expected that NCED-type activity and expression of OsNCED genes should also be upregulated by coumarin. Interestingly, however, coumarin did not substantially increase OsNCED activity or content, or OsNCED transcription. Conversely, OsNCED gene expression and enzymatic activity were downregulated at certain imbibition times (Figures 4, 6). These results were confirmed by showing that the percentage of germinated rice seeds that had been imbibed in coumarin and the ABA-synthesis inhibitor, fluridone, was not increased compared with seeds imbibed in coumarin alone (Supplementary Figure S1).

Coumarin significantly inhibited the expression of OsABA8'ox $2 / 3$ in the ABA catabolic pathway (Figures 7E,F). The low-level expression of these genes led to decreased OsABA8'ox activity and, consequently, a decreased rate of ABA degradation and increased ABA accumulation in comparison with water-imbibed seeds. These results are consistent with those showing that the $\mathrm{ABA}$ content was relatively increased 

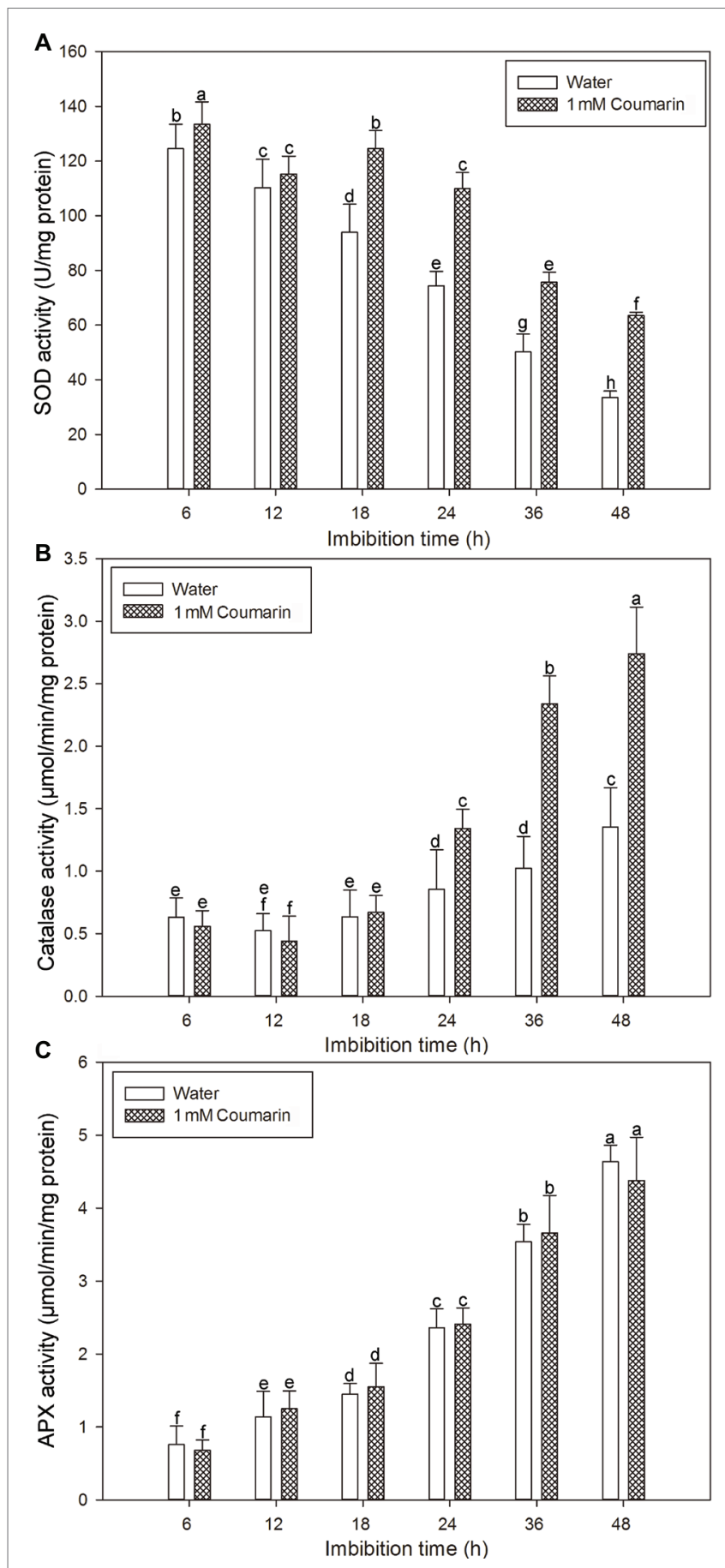

FIGURE 8 | Changes in the activities of ROS-degrading enzymes in rice seeds. Effects of coumarin on the activity of SOD (A), catalase (B), and APX (C) in embryos assayed via spectrophotometry after their seeds had been imbibed in water or $1 \mathrm{mM}$ coumarin for $6,12,18,24,36$, or $48 \mathrm{~h}$. Data represent the mean \pm SE of three biological replicates, each with 150 embryos ( $0.5 \mathrm{~g}$ per replicate). Means denoted by the same letter did not significantly differ at $p<0.05$ according to Fisher's least significant difference test.

in coumarin-imbibed seeds in comparison with those imbibed in water (Figure 3). In addition, in the presence of coumarin and diniconazole, seed germination was substantially inhibited compared with germination of seeds that were coumarin imbibed (Supplementary Figure S1). Given the above discussion, we believe that inhibition of rice seed germination may be a consequence of inhibition of ABA catabolism by coumarin. Consequently, coumarin may inhibit rice germination by upregulating expression of $O s A B A 8^{\prime} \circ \times 2 / 3$, thereby increasing the ABA content. Our results are consistent with the observations that copper and glucose inhibit seed germination by suppressing ABA catabolism (Zhu et al., 2009; Ye et al., 2014).

\section{Coumarin May Regulate Endogenous Abscisic Acid Content and Reactive Oxygen Species Accumulation}

Numerous studies have shown that an excessive accumulation of ROS is the underlying cause of oxidative stress in plants (Sharma et al., 2012). ROS also serve as signaling molecules during plant growth and in the development and responses to biotic and abiotic stresses (Fujita et al., 2006). Recently, however, ROS had been shown to directly act on plant cellwall polysaccharides by breaking their glycosidic bonds, which cause the cell wall to relax, thereby promoting cell division and elongation (Zhang et al., 2014). During seed germination of dicotyledonous plants, such as cress and lettuce, ROS accumulate in their micropylar endosperm and radicle, led to endosperm weakening and radicle elongation, thereby promoting seed germination (Müller et al., 2009). Certain cell-wall hydrolases and non-enzymatic substances, such as ROS and expansin, are needed for these processes (GonzálezCalle et al., 2015). We previously found that ROS accumulated in the radicle and coleorhiza of rice seeds during germination and that ROS production and the number of germinated seeds decreased in the presence of ROS inhibitors, i.e., diphenyleneiodonium chloride and guazatine (Li et al., 2017). Therefore, for this study, we also wanted to explore the effect(s) of coumarin on ROS accumulation in germinating rice seeds. For the coumarin-imbibed seeds, less ROS, as $\mathrm{O}_{2}^{-}$and $\mathrm{H}_{2} \mathrm{O}_{2}$, accumulated in embryos than in the embryos from waterimbibed seeds, although the peroxidase activity was not substantially affected, indicating that the $\bullet \mathrm{OH}$ concentration was not much affected. These results indicated that $\mathrm{O}_{2}^{-}$and $\mathrm{H}_{2} \mathrm{O}_{2}$ might be the main types of ROS involved in germination. Seed viability can affect ROS accumulation in seeds to a certain extent. For example, when lettuce seeds were imbibed in a $0.3 \%$ sodium dichloroisocyanurate solution, the observed decrease in ROS accumulation may have been caused by a decrease in the micropylar endosperm and radicle viability (Zhang et al., 2014). We found that coumarin did not reduce rice embryo viability (Figure $\mathbf{7 A}$ ), indicating that coumarin may not inhibit the germination of rice seeds and that the inhibition of ROS accumulation cannot be achieved by reducing the viability of the rice seed embryo. SOD, catalase, and APX are ROS-degrading enzymes and are involved in the regulation of ROS accumulation in plants (Ye et al., 2014). Coumarin increased the SOD and catalase activities, especially at the later stage of seed imbibition, but had no substantial effect on APX activity (Figures 8A,B), indicating that coumarin may 
specifically enhance the expression of SOD and catalase, thereby accelerating ROS degradation in the embryo. However, the mechanism remains to be further studied.

There is crosstalk between ROS and phytohormones in seed germination. ROS production is increased by ABA in stressed plants (Gechev et al., 2006), but the regulatory role of ABA on ROS production differs between seed and non-seed tissues (Ye et al., 2012). During seed germination of cress and rice, ABA causes a decrease in $\cdot \mathrm{OH}$ radical production in seeds (Müller et al., 2009). In Hedysarum scoparium seeds, ABA treatment significantly inhibited germination and reduced the $\mathrm{H}_{2} \mathrm{O}_{2}$ content in both cold-stratified and non-cold-stratified seeds (Su et al., 2016). In our present study, coumarin, which inhibits germination, increased the ABA content while decreasing the ROS content of the embryo. In conclusion, our results enhance our understanding of the regulation of ABA and ROS levels during seed germination, although the underlying mechanisms need further study. Furthermore, our research will probably contribute to farming, such as the use of coumarin as a practical compound to prevent viviparous germination of crops.

\section{DATA AVAILABILITY}

All datasets generated for this study are included in the manuscript and/or the Supplementary Files.

\section{AUTHOR CONTRIBUTIONS}

JL, B-XC, and HF designed the research. B-XC, Y-XP, J-DG, $\mathrm{QZ}$, and Q-JL performed the experiments. B-XC analyzed the data and wrote the manuscript. JL and B-XC critically revised the manuscript.

\section{REFERENCES}

Ali-Rachedi, S., Bouinot, D., Wagner, M.-H., Bonnet, M., Sotta, B., Grappin, P., et al. (2004). Changes in endogenous abscisic acid levels during dormancy release and maintenance of mature seeds: studies with the Cape Verde Islands ecotype, the dormant model of Arabidopsis thaliana. Planta 219, 479-488. doi: 10.1007/s00425-004-1251-4

Batty, A. L., Dixon, K. W., Brundrett, M., and Sivasithamparam, K. (2001). Long-term storage of mycorrhizal fungi and seed as a tool for the conservation of endangered Western Australian terrestrial orchids. Aust. J. Bot. 49, 619-628. doi: 10.1071/BT01029

Berrie, A. M. M., Parker, W., Knights, B. A., and Hendrie, M. R. (1968). Studies on lettuce seed germination-I. Coumarin induced dormancy. Phytochemistry 7, 567-573. doi: 10.1016/S0031-9422(00)88228-X

Bewley, J. D., Bradford, K. J., Hilhorst, H. W. M., and Nonogaki, H. (2013). Seeds: Physiology of development, germination and dormancy. (New York, NY: Springer-Verlag).

Bradford, M. M. (1976). A rapid and sensitive method for the quantitation of microgram quantities of protein utilizing the principle of protein-dye binding. Anal. Biochem. 72, 248-254. doi: 10.1016/0003-2697(76)90527-3

Chang, Y. L., Li, W. Y., Miao, H., Yang, S. Q., Li, R., Wang, X., et al. (2016). Comprehensive genomic analysis and expression profiling of the NOX gene families under abiotic stresses and hormones in plants. Genome Biol. Evol. 8, 791-810. doi: 10.1093/gbe/evw035

\section{FUNDING}

This work was supported by the Natural Science Foundation of China (Grant No. 31601388 and 31871716), the Natural Science Foundation of Guangdong Province (Grant No. 2016A030310320), the Foundation of President of Guangdong Academy of Agricultural Sciences (Grant No. 201724), and the Science and Technology Planning Project of Guangdong Province (2016B030303007 and 2018B020202004).

\section{SUPPLEMENTARY MATERIAL}

The Supplementary Material for this article can be found online at: https://www.frontiersin.org/articles/10.3389/fpls.2019.00828/ full\#supplementary-material

SUPPLEMENTARY FIGURE S1 | Effect of coumarin, ABA biosynthesis inhibitor, and (or) ABA catabolic inhibitor on the germination percentage of rice seeds. Data represent means \pm SE of three biological replicates of 100 seeds each. Means denoted by the same letter did not significantly differ at $p<0.05$ according to Fisher's least significant difference test.

SUPPLEMENTARY FIGURE S2 | Expression profiles for rice OsNCED1-5 and OsABA8'ox2/3 genes. Expression profiles, shown as heat maps, for rice OsNCED1-5 and OsABA8'ox2/3 genes during the different periods of rice growth (A) and seed germination (B). Expression profiles were obtained from rice microarray data (Os_51k array) as reported in Genevestigator V3. Expression profiles for OsABA8'ox1 were unavailable. The white/blue (A) and green/red (B) colors reflect the relative expression levels, with dark green representing strong downregulation and dark red representing strong upregulation.

SUPPLEMENTARY TABLE S1 | ABA metabolism-related genes in Oryza sativa, Arabidopsis thaliana, and other plant species.

SUPPLEMENTARY TABLE S2 | Primer sequences for qPCR of OsGAPDH1, OsNCEDs, and OSABA8'OXs.

Chen, B., Ma, J., Xu, Z., and Wang, X. (2016). Abscisic acid and ethephon regulation of cellulase in the endosperm cap and radicle during lettuce seed germination. J. Integr. Plant Biol. 58, 859-869. doi: 10.1111/jipb.12479

Colpas, F. T., Ono, E. O., Rodrigues, J. D., and Passos, J. R. D. S. (2003). Effects of some phenolic compounds on soybean seed germination and on seed-borne fungi. Braz. Arch. Biol. Technol. 46, 155-161. doi: 10.1590/ S1516-89132003000200003

Durantini, D., Giulini, A., Malgioglio, A., Pilu, R., Tuberosa, R., Sanguineti, C., et al. (2008). Vivipary as a tool to analyze late embryogenic events in maize. Heredity 101, 465-470. doi: 10.1038/hdy.2008.29

Frey, A., Effroy, D., Lefebvre, V., Seo, M., Perreau, F., and Berger, A. (2012). Epoxycarotenoid cleavage by NCED5 fine-tunes ABA accumulation and affects seed dormancy and drought tolerance with other NCED family members. Plant J. 70, 501-512. doi: 10.1111/j.1365-313x.2011.04887.x

Fujita, M., Fujita, Y., Noutoshi, Y., Takahashi, F., Narusaka, Y., YamaguchiShinozaki, K., et al. (2006). Crosstalk between abiotic and biotic stress responses: a current view from the points of convergence in the stress signaling networks. Curr. Opin. Plant Biol. 9, 436-442. doi: 10.1016/j. pbi.2006.05.014

Gechev, T. S., Van Breusegem, F., Stone, J. M., Denev, I., and Laloi, C. (2006). Reactive oxygen species as signals that modulate plant stress responses and programmed cell death. BioEssays 28, 1091-1101. doi: 10.1002/bies.20493

González-Calle, V., Barrero-Sicilia, C., Carbonero, P., and Iglesias-Fernández, R. (2015). Mannans and endo- $\beta$-mannanases (MAN) in brachypodiumdistachyon: 
expression profiling and possible role of the BdMAN genes during coleorhizalimited seed germination. J. Exp. Bot. 66, 3753-3764. doi: 10.1093/jxb/erv168

Hruz, T., Laule, O., Szabo, G., Wessendorp, F., Bleuler, S., Oertle, L., et al. (2008). Genevestigator v3: a reference expression database for the metaanalysis of transcriptomes. Adv. Bioinforma. 2008:420747. doi: 10.1155/2008/420747

Khan, A. A., and Tolbert, N. E. (1966). Light-controlled cycocel reversal of coumarin inhibition of lettuce seed germination and root growth. Physiol. Plant. 19, 76-80. doi: 10.1111/j.1399-3054.1966.tb09076.x

Kitahata, N., Saito, S., Miyazawa, Y., Umezawa, T., Shimada, Y., Yong, K., et al. (2005). Chemical regulation of abscisic acid catabolism in plants by cytochrome P450 inhibitors. Bioorg. Med. Chem. 13, 4491-4498. doi: 10.1016/j. bmc.2005.04.036

Koornneef, M., Léon-Kloosterziel, K. M., Schwartz, S. H., and Zeevaart, J. A. D. (1998). The genetic and molecular dissection of abscisic acid biosynthesis and signal transduction in Arabidopsis. Plant Physiol. Biochem. 36, 83-89. doi: 10.1016/S0981-9428(98)80093-4

Kushiro, T., Okamoto, M., Nakabayashi, K., Yamagishi, K., Kitamura, S., and Asami, T. (2004). The Arabidopsis cytochrome P450 CYP707A encodes ABA 8'-hydroxylases: key enzymes in ABA catabolism. EMBO J. 23, 1647-1656. doi: 10.1038/sj.emboj.7600121

Li, W.-Y., Chen, B.-X., Chen, Z.-J., Gao, Y.-T., Chen, Z., and Liu, J. (2017). Reactive oxygen species generated by $\mathrm{NADPH}$ oxidases promote radicle protrusion and root elongation during rice seed germination. Int. J. Mol. Sci. 18:110. doi: 10.3390/ijms18010110

Liu, F., Zhang, H., Wu, G., Sun, J., Hao, L., Ge, X., et al. (2011). Sequence variation and expression analysis of seed dormancy-and germination-associated ABA- and GA-related genes in rice cultivars. Front. Plant Sci. 2:17. doi: 10.3389/fpls.2011.00017

Martínez-Andújar, C., Ordi, M. I., Huang, Z., Nonogak, M., Beach, R. N., and Nonogak, H. (2011). Induction of 9-cis-epoxycarotenoid dioxygenase in Arabidopsis thaliana seeds enhances seed dormancy. PNAS 108, 17225-17229. doi: $10.2307 / 41321860$

Matakiadis, T., Alboresi, A., Jikumaru, Y., Tatematsu, K., Pichon, O., and Renou, J.-P. (2009). The Arabidopsis abscisic acid catabolic gene CYP707A2 plays a key role in nitrate control of seed dormancy. Plant Physiol. 149, 949-960. doi: 10.1104/pp.108.126938

Millar, A. A., Jacobsen, J. V., Ross, J. J., Helliwell, C. A., Poole, A. T., Scofield, G., et al. (2006). Seed dormancy and ABA metabolism in Arabidopsis and barley: the role of ABA 8'-hydroxylase. Plant J. 45, 942-954. doi: 10.1111/j. 1365-313x.2006.02659.x

Müller, K., Hess, B., and Leubner-Metzger, G. (2007). "A role for reactive oxygen species in endosperm weakening" in Seeds: Biology, development and ecology. eds. S. Adkins, S. Ashmore, and S. Navie (Wallingford, UK: CAB International), 287-295.

Müller, K., Linkies, A., Vreeburg, R. A., Fry, S. C., Krieger-Liszkay, A., and LeubnerMetzger, G. (2009). In vivo cell wall loosening by hydroxyl radicals during cressseed germination and elongation growth. Plant Physiol. 150, 1855-1865. doi: 10.1104/pp.109.139204

Nambara, E., and Marion-Poll, A. (2005). Abscisic acid biosynthesis and catabolism. Annu. Rev. Plant Biol. 56, 165-185. doi: 10.1007/978-94-009-3585-3_7

Nonogaki, H., Barrero, J. M., and Li, C. (2018). Seed dormancy, germination and pre-harvest sprouting. Front. Plant Sci. 9:1783. doi: 10.3389/fpls.2018.01783

Nonogaki, M., Sall, K., Nambara, E., and Nonogaki, H. (2014). Amplification of ABA biosynthesis and signaling through a positive feedback mechanism in seeds. Plant J. 78, 527-539. doi: 10.1111/tpj.12472
Qin, X., and Zeevaart, J. A. D. (1999). The 9-cis-epoxycarotenoid cleavage reaction is the key regulatory step of abscisic acid biosynthesis in waterstressed bean. PNAS 96, 15354-15361. doi: 10.1073/pnas.96.26.15354

Robichaud, C. S., Wong, J., and Sussex, I. M. (1980). Control of in vitro growth of viviparous embryo mutants of maize by abscisic acid. Dev. Genet. 1, 325-330. doi: 10.1002/dvg.1020010405

Rodríguez-Gacio, M. D. C., Matilla-Vázquez, M. A., and Matilla, A. J. (2009). Seed dormancy and ABA signaling: the breakthrough goes on. Plant Signal. Behav. 4, 1035-1049. doi: 10.1111/j.1365-3040.2012.02542.x

Saika, H., Okamoto, M., Miyoshi, K., Kushiro, T., Shinoda, S., Jikumaru, Y., et al. (2007). Ethylene promotes submergence-induced expression of OsABA80x1, a gene that encodes ABA 8'-hydroxylase in rice. Plant Cell Physiol. 48, 287-298. doi: 10.1093/pcp/pcm003

Sharma, P., Jha, A., Dubey, R., and Pessarakli, M. (2012). Reactive oxygen species, oxidative damage, and antioxidative defense mechanism in plants under stressful conditions. Aust. J. Bot. 2012, 1-26. doi: 10.1155/2012/217037

Su, L., Lan, Q., Pritchard, H., Xue, H., and Wang, X. (2016). Reactive oxygen species induced by cold stratification promote germination of Hedysarum scoparium seeds. Plant Physiol. Biochem. 109, 406-415. doi: 10.1016/j. plaphy.2016.10.025

Wang, J. Y., Yao, D. D., Xu, C. X., Zhao, G. Q., and Hua, C. L. (2017). Effect of coumarin on sorghum sudanense seed germination and seedling growth. Pratacult. Sci. 34, 2279-2288. doi: 10.11829/j.issn.1001-0629.2017-0008

Yamaguchi, S., Kamiya, Y., and Nambara, E. (2007). "Regulation of ABA and GA levels during seed development and germination in Arabidopsis" in Seed development, dormancy and germination. Annual plant reviews (Vol. 27). eds. K. J. Bradford, and H. Nonogaki (Oxford: Blackwell Publishing), 224-247.

Ye, N., Li, H., Zhu, G., Liu, Y., Liu, R., Xu, W., et al. (2014). Copper suppresses abscisic acid catabolism and catalase activity, and inhibits seed germination of rice. Plant Cell Physiol. 55, 2008-2016. doi: 10.1093/pcp/pcu136

Ye, N., Zhu, G., Liu, Y., Zhang, A., Li, Y., and Liu, R. (2012). Ascorbic acid and reactive oxygen species are involved in the inhibition of seed germination by abscisic acid in rice seeds. J. Exp. Bot. 63, 1809-1822. doi: 10.1093/jxb/ err336

Zhang, Y., Chen, B., Xu, Z., Shi, Z., Chen, S., Huang, X., et al. (2014). Involvement of reactive oxygen species in endosperm cap weakening and embryo elongation growth during lettuce seed germination. J. Exp. Bot. 65, 3189-3200. doi: $10.1093 /$ jxb/eru167

Zhu, G., Ye, N., and Zhang, J. (2009). Glucose-induced delay of seed germination in rice is mediated by the suppression of $\mathrm{ABA}$ catabolism rather than an enhancement of ABA biosynthesis. Plant Cell Physiol. 50, 644-651. doi: $10.1093 / \mathrm{pcp} / \mathrm{pcp} 022$

Conflict of Interest Statement: The authors declare that the research was conducted in the absence of any commercial or financial relationships that could be construed as a potential conflict of interest.

Copyright (c) 2019 Chen, Peng, Gao, Zhang, Liu, Fu and Liu. This is an open-access article distributed under the terms of the Creative Commons Attribution License (CC BY). The use, distribution or reproduction in other forums is permitted, provided the original author(s) and the copyright owner(s) are credited and that the original publication in this journal is cited, in accordance with accepted academic practice. No use, distribution or reproduction is permitted which does not comply with these terms. 